\section{P-210 BRINGING END OF LIFE CARE TO PATIENTS THROUGH HOSPICE OUTPATIENT CLINICS}

Angie Redpath, Jude Richardson, Ann Smith. Princess Alice Hospice, Esher, UK

10.1136/bmjspcare-2019-HUKNC.232

Background The End of Life Care strategy recommended the delivery of high quality services for patients needing palliative care, access to rapid, specialist advice and clinical assessment (Department of Health, 2008).

Clinics run by Clinical Nurse Specialists (CNSs) are at the forefront of modernising the health service and ensuring that CNSs have the ability to influence and lead in advanced practice. There is a paucity of evidence to support such clinics in palliative care, however, feedback from patients and families tell us they are interested in attending local outpatient clinics.

Aim As part of the hospice strategy to reach more people, a project to deliver local outpatient clinics was developed with the aim of improving efficiency and coordination of services in our community.

Method Four outpatient clinics were established across the hospice's catchment area, led by the Nurse Consultant. Good working relationships with local General Practitioners and commissioners allowed for the provision of the clinic space.

Results There was some initial resistance. However, increased choice for patients, improved multi-professional working with primary healthcare professionals, and reducing the CNSs workload has supported the development of outpatient clinics.

$42 \%$ patients remained on the outpatient clinics caseload, $53 \%$ were referred on to the Hospice at Home team, 2\% attended Day services and 3\% were discharged.

$7 \%$ had a non-cancer diagnosis, this may reflect the lateness of referrals, and are too unwell to attend outpatient clinics.

Medication changes were recommended in $60 \%$ of patients, Advance Care Planning with $93 \%$ and liaison with acute services in $60 \%$ of patients.

Conclusions Outpatient clinics can alleviate pressure on the CNS team, set the direction of travel for patients as well as proactively managing patients' concerns, symptoms and future plans.

On-going evaluation will continue to demonstrate how these clinics can be developed to support the challenges faced with increased demand on limited resources.

\section{P-211 A BLUE LIGHT PARTNERSHIP: INNOVATIVE COLLABORATION BETWEEN AN AMBULANCE SERVICE AND HOSPICE}

${ }^{1}$ Georgina Murphy-Jones, ${ }^{2}$ Charles Daniels, ${ }^{1}$ Karina Catley, ${ }^{1}$ Diane Laverty, ${ }^{1}$ Caroline Phillips, ${ }^{2}$ Inderia Tipping. 'London Ambulance Service NHS Trust, London, UK; ${ }^{2}$ St Luke's Hospice (Harrow and Brent) London, UK

\subsection{6/bmjspcare-2019-HUKNC.233}

Background A Metropolitan Ambulance Service has partnered with Macmillan Cancer Support on a two year programme to improve end-of-life care. This aims to reduce inappropriate hospital conveyance and increase staff knowledge and confidence; when surveyed $50 \%$ of ambulance clinicians 'agreed' or 'strongly agreed' with feeling confident in their skills and knowledge of end-of-life care.
Aims Evaluate the impact of a co-production programme on ambulance clinicians' knowledge and confidence in responding to patients in the last phase of life. Inform development of the Ambulance Service's end-of-life care strategy.

Methods An interested hospice was invited to co-design and co-deliver an interactive CPD event for ambulance clinicians. Paramedic End-of-Life Care Champions were supported by the ambulance service Macmillan team, Hospice Consultant and hospice telephone helpline nurse manager. The event was evaluated via online survey and staff interviews captured learning which may facilitate spread of hospice co-production service wide.

Results Results evidence the programme's success:

- 34 operational ambulance staff attended;

- $100 \%$ agreed or strongly agreed that it positively influenced their practice:

- Increased confidence in skills and knowledge of end-of-life care, $78 \%$ respondents agreed/strongly agreed with feeling confident.

- $47 \%$ reported learning about hospice services which they could use in practice;

- Case based discussions were unanimously regarded as an excellent method of learning, with palliative care expertise valued;

- Hospice staff unexpectedly learnt important lessons from ambulance colleagues which will improve their service.

Conclusions Alongside learning for ambulance clinicians there was unintended but beneficial learning for hospice staff. The hospice and Macmillan teams provided essential support to the Paramedic End-of-Life Care Champions but the production of a 'How to run an Interactive end-of-life care training event' kit would reduce the need for central support. The benefits to hospices in utilisation of, and improvement in their services and their staff may help encourage wider support from hospices to be involved in similar co-production events.

\section{P-212 THE DESIGN AND IMPLEMENTATION OF THE HOSPICE NIGHT SUPPORT TEAM}

Liz Morgan. Nottinghamshire Hospice, Nottingham, UK

10.1136/bmispcare-2019-HUKNC.234

The Hospice Night Support Service was designed to complement and support our Hospice at Home service and increase palliative care to patients in their preferred place of death. Unlike Hospice at Home, not all patients required a nurse to be in their home all night. It therefore increased capacity in this service while at the same time providing shorter term care to more patients.

It was decided that a responsive overnight visiting service could prevent hospital admission and speed up discharge of patients out of hospital to their homes. District nursing capacity challenges them to provide urgent care only overnight rather than personal and emotional care. This new service was designed to provide care interventions such as mouth care, continence care, and emotional support and advice to patients and carers when they need it most.

Patients and carers can either pre-book or phone for a visit at the time they need care. Implementation included increasing 
skills of the workforce, liaising with key referrers and securing funding.

Throughout the first complete month the service made 200 visits, half of these provided emotional support. The team have been called by patients who previously frequently called 999 when they were anxious or distressed, hence now freeing up a much more costly service.

45 of the visits were to provide continence care to patients, one relative said 'my father would have suffered through the night as I struggle to clean him myself'.

The current Hospice at Home service is a limited resource providing care to people in the last days of life. Carers are satisfied with a short visit from the Hospice Night Support Team when a whole Hospice at Home shift is unavailable. This is an excellent way of supporting family and patients in their preferred place of care while efficiently using resources.

\section{P-213 WILLEN AT HOME AN INTEGRATED COMMUNITY SERVICE}

Anita Connolly, Jackie Meissner. Willen Hospice, Milton Keynes, UK

\subsection{6/bmjspcare-2019-HUKNC.235}

Background The Department of Health End of Life Care Strategy (2008) identified an increasing need for good end of life care in the community. The planning and reorganisation of this new community service has been developed to meet the demands of managing patients with more complex disease trajectories in an ever growing local population.

The new team Willen at Home is a new integrated community service, which combines the original Hospice at Home and Clinical Nurse Specialist Teams CNSs). The Willen at Home team was established in January 2019.The vision for this new team was to provide a holistic and responsive service using the combined skills of a multidisciplinary team. Patients are triaged according to their Phase of Illness and Performance Status (AKPS, 2005), and care is provided by the skill mix of professionals appropriate to their needs. Unwell, unstable or dying patients are triaged, ensuring a timely response as their condition changes. The integrated team comprises: Community Lead Nurse, CNS, Community Senior Nurse, Registered Nurses, Healthcare Assistants, Doctors.

Each day, staff are assigned to core roles:

- Telephone triage;

- Visiting;

- Monitoring.

This enables prompt assessment, prescribing, delivery of care and liaison with secondary care.

Impact of the new team In the new team, staff have a designated role for the day, ensuring that triage, assessment, visiting, prescribing and care planning are carried out in a personalised and timely way. As a result of this: Multidisciplinary team working means the patient's care is tailored to their needs on the day. Clinical staff work collaboratively with allied services such as Social Work, Psychology and Patient and Family services. Nurse prescribers provide timely access to specialist medications. Patients can be reviewed on the day by a specialist doctor. More patients receive specialist care at home and are enabled to die in their own surroundings, avoiding unwanted or crisis admissions.

\section{P-214 HOSPICE AT HOME RESPITE SERVICE}

Debby Varnam. St. Giles Hospice, Lichfield, UK

\subsection{6/bmispcare-2019-HUKNC.236}

Recently the organisation has been looking at how to improve support for our patients and their families in the community. Our already well established Hospice at Home service offers personal care, respite support and night sits for patients in their last few weeks of life. However, the organisation recognised that there was still more needed to enable families and patients to feel supported at home. After speaking to families, patients and community healthcare professionals, including GPs and District Nurses, the organisation identified a gap in service provision for respite care. Consequently, in January this year we launched our new respite service.

The aim of this new service is to provide support to patients and carers in the last 12 months of life by providing respite care within the home setting. The service aims to support family members caring for relatives with a life limiting illness which can result in physical and emotional problems directly related to the stress and demands of daily care. By offering much needed respite the aim is that this will enable patients to remain at home longer and avoid unwanted admissions to other health care settings.

Everyone needs some time for themselves, even more so when they care for someone else. Respite has traditionally been offered as residential, however by offering respite care in the home this causes minimal disruption to the patient and provides the carer with a much needed break. The service aims to be as flexible as possible offering visits during the morning, afternoon or early evening, seven days per week. This gives carers the opportunity to attend appointments, have a much needed rest, or time out to enjoy social activities.

We continually evaluate the service by gathering feedback from our service user surveys. The feedback we have received has been extremely positive and reflects how much this service is needed.

\section{P-215 DEVELOPING A LIVING SUPPORT GROUP - LEARNING FROM PATIENT FEEDBACK}

Dympna Jones, Katherine Rugen, Kate McIntegart. Woodlands Hospice, Liverpool, UK

10.1136/bmjspcare-2019-HUKNC.237

Background Woodlands Hospice has run a 'Supportive Living Programme' aiming to enable patients to live as well as they can, for as long as they can, for several years. This is a 10 week rolling programme of clinician-led sessions on varied topics relating to patient wellbeing, which is well attended and receives positive feedback. Following completion of this programme, patients are encouraged to attend an informal peer support group where they can share experiences, make friends and grow in confidence. Over time it became apparent that attendance at this group was diminishing and feedback from the patients was that they missed the clinical support they had enjoyed in the 10 week programme.

Aims The hospice was keen to ensure ongoing appropriate support and enable patients to feel safe in the knowledge 